\title{
Nonlocal transport through multiterminal diffusive superconducting nanostructures
}

\author{
F. S. Bergeret \\ Departamento de Física Teórica de la Materia Condensada C-V, Facultad de Ciencias, Universidad Autónoma de Madrid, \\ E-28049 Madrid, Spain; \\ Centro de Física de Materiales CFM-MPC, CSIC-UPV/EHU, Edificio Korta, Avenida de Tolosa 72, \\ 20018 San Sebastián, Spain; \\ and Donostia International Physics Center (DIPC), Manuel de Lardizábal 4, 20018 San Sebastián, Spain \\ A. Levy Yeyati \\ Departamento de Física Teórica de la Materia Condensada C-V, Facultad de Ciencias, Universidad Autónoma de Madrid, \\ E-28049 Madrid, Spain
}

(Received 7 August 2009; published 9 November 2009)

\begin{abstract}
Motivated by recent experiments on nonlocal transport through multiterminal superconducting hybrid structures, we present self-consistent calculations based on quasiclassical Green's functions for the order parameter, currents, and voltages in a system consisting of a diffusive superconductor connected to two normal and one superconducting electrodes. We investigate nonequilibrium effects for different biasing conditions corresponding to measurements of the nonlocal conductance and of the nonlocal resistance. It is shown that while the nonlocal conductance does not change its sign, this change might be observed in a nonlocal resistance measurement for certain parameter range. The change in sign of the nonlocal signal takes places at a voltage of the order of the self-consistent gap of the superconducting region. We show that this is not related to the nonlocal Andreev processes but rather to nonequilibrium effects. We finally discuss the case of four terminal measurements and demonstrate that a change in sign in the nonlocal resistance appears when the current injected into the superconductor exceeds a critical value. The connection to the existing experiments is discussed.
\end{abstract}

DOI: $10.1103 /$ PhysRevB.80.174508

PACS number(s): 74.45.+c, 74.78.Fk, 74.50.+r

\section{INTRODUCTION}

The possibility to create and control entangled electron pairs from a superconductor has renewed the interest in transport through superconductor-normal metal hybrid structures. ${ }^{1-5}$ The basic idea is to exploit the long-range coherence of the Andreev reflection, ${ }^{6}$ in order to couple two spatially separated normal electrodes connected to a superconducting region within a distance of the order of the superconducting coherence length $\xi_{S}$. An Andreev processes that takes place at two different interfaces is called a "crossed" Andreev reflection (CAR). As suggested in Ref. 7 spatial correlations can be probed by nonlocal transport experiments. A typical setup for detection of CAR processes consists of a grounded superconducting (S) region connected to normal $(\mathrm{N})$ electrodes. The information on such processes would be encoded, for instance, in the voltage which is measured in one of the $\mathrm{S} / \mathrm{N}$ interfaces when a current is injected through the other one. Besides CAR processes, individual electrons can also tunnel across the superconductor. This normal tunneling has been called "elastic cotunneling" (EC). CAR and EC contributions to the nonlocal conductance have opposite signs, and in the lowest order of tunneling cancel each other. ${ }^{8}$ For higher orders in the tunneling the EC dominates over CAR (Ref. 9) and the nonlocal signal becomes finite. Surprisingly a change in sign in the nonlocal resistance and conductance was reported as a function of the local voltage for a NSN layered structure ${ }^{2}$ and a FSF multiterminal structure $^{4}$ ( $\mathrm{F}$ denotes a ferromagnetic metal). According to Ref. 2 by low (high) voltages EC (CAR) processes dominate the nonlocal transport. Similar behavior was observed in Ref. 4 for samples with high S/F barrier resistance. The latter experiment also showed dominance of CAR processes at low voltages for samples with higher interface transparency. These experimental results have lead to several theoretical works, ${ }^{9-17}$ which attempt to find a microscopic description for those observations. However, up to now theories based on noninteracting models could not explain the change in sign of the nonlocal conductance. ${ }^{10-13}$ For a layered NSN structure, as the one of Ref. 2, the change in sign of the nonlocal conductance has been explained by taking into account interaction of the conducting electrons with their electromagnetic environment. ${ }^{16}$ The later description is valid in the tunneling limit, thus the observation of negative nonlocal conductances in the case of good interface transparencies remains without microscopic explanation yet.

In principle, a description of the nonlocal transport in terms of CAR and EC is only valid in the tunneling limit. In this case the system is in a quasiequilibrium state, i.e., the current (or the corresponding bias voltage) is much smaller than its critical value and the distribution function of quasiparticles is the equilibrium one. This assumption has been made in most of the theoretical works mentioned above. In particular the superconducting gap was assumed to have the bulk value, i.e., the superconducting order parameter was not affected by the possible deviation of the distribution function from its equilibrium value. In some experiments though, this is not the case. For example in Refs. 1, 3, and 5 the transparencies of the $\mathrm{S} / \mathrm{N}$ interfaces are not necessarily low, and in Ref. 3 the current injected into the $S$ region reached its critical value. Thus, for a proper description of these experiments one needs to go beyond the quasiequilibrium approach. A first attempt was done in Refs. 14 and 15. It was shown that nonequilibrium effects may play a crucial role on 
the nonlocal transport properties. In particular, in Ref. 14 self-consistent calculations based on a two-dimensional tight-binding model were implemented. It was shown that far from the quasiequilibrium regime the nonlocal transport cannot longer be described in simple terms of EC and CAR processes. For some set of parameters, a change in sign in the nonlocal resistance was obtained. This change in sign is not related to the predominance of CAR but rather to the possibility of having a negative local conductance at the interface where the current is injected. In Ref. 15 a nonmonotonic behavior of the nonlocal resistance as a function of the temperature was obtained, which resembles the observations of Ref. 3. However, the nonlocal resistance as a function of the injected current or bias voltage was not investigated in that work.

In the above-mentioned theoretical works, one computes the nonlocal conductance in a three-terminal device. In other words, one assumes that one of the normal terminals is biased to a voltage $V_{L}$, while the second normal terminal is grounded. In this way one determines the current $I_{R}$ flowing into the latter terminal and computes the nonlocal conductance $G_{\mathrm{nl}}=d I_{R} / d V_{L}$. Experimentally, however, it is simpler to fix the injected current through one of the normal terminals and measure the voltage induced at the second normal terminal where no current is flowing. Thus, the measured quantity is the nonlocal resistance. Moreover, some experiments were performed in a multiterminal geometry. ${ }^{3}$

In this paper we present a complete self-consistent theory for the nonlocal transport through a diffusive superconducting region connected to several normal electrodes. We calculate both, the nonlocal conductance and resistance with the help of the quasiclassical Green's function (GF) approach. In a first part we concentrate on a three-terminal device, where the current is injected from a normal electrode $N_{L}$ into the superconducting region $S$, maintaining the second normal electrode $N_{R}$ grounded. We determine the self-consistent gap, the current flowing into $N_{R}$ and compute the nonlocal conductance $G_{\mathrm{nl}}$. We show that our model, as in previous works, predicts no change in sign for $G_{\mathrm{nl}}$. In a second part we consider again a three-terminal device, but now we assume that no current is flowing at the $S / N_{R}$ interface terminal. We then determine the self-consistent gap, the voltage induced in $N_{R}$, and determine the nonlocal resistance $R_{\mathrm{nl}}$. For certain range of parameters we obtain a change in sign $R_{\mathrm{nl}}$ due to the appearance of a negative local conductance at the $N_{L} / S$ interface. Finally, we considered a four terminal setup. Again we assume that the current through the $S / N_{R}$ interface is zero, but now we determine the voltage induced in the $N_{R}$ electrode measured with respect to the end of the superconductor in which no current is flowing. In this case we obtain a change in sign as in the experiment of Ref. 3. As we show below the origin of this change in sign is not due to a negative local conductance, but to the nonequilibrium distribution created in the superconductor by the injected current, which eventually leads to a transition into the normal state.

The rest of the paper is organized as follows: in Sec. II we introduce the model and basic equations used throughout the paper, Secs. III and IV are devoted to analyze the results for the three-terminal situation (we discuss the conductance measurement conditions in Sec. III and the resistance mea-

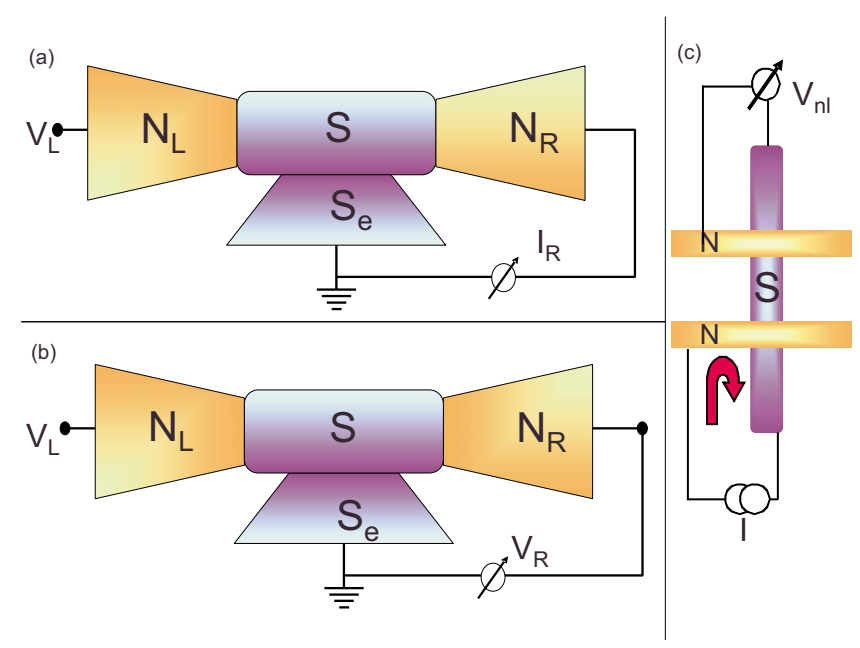

FIG. 1. (Color online) Sketch of a three-terminal device for (a) measurement of the nonlocal conductance and (b) measurement of the nonlocal resistance. (c) Typical experimental setup for measurement of the nonlocal resistance.

surement case in Sec. IV), and finally in Sec. V we analyze the four terminal case. Some concluding remarks are given in Sec. VI.

\section{MODEL AND BASIC EQUATIONS}

A typical experimental setup for the measurement of nonlocal transport properties is shown in Fig. 1(c). On top of a nanoscale superconducting wire one places several (in our case two) normal wires. A current is injected from one of the normal electrodes and flows in the direction shown in the figure. The nonlocal resistance is then obtained by measuring the potential difference between the other normal electrode and the end of the superconducting wire through which no current is flowing. A strong enough nonlocal signal is measured when the distance between the normal leads does not exceed much the coherence length of the superconductor.

A self-consistent computation of the spatial variation in the gap and currents in such a device is a formidable task which we will not address here. Nevertheless, the mean features of the system can be described by considering the geometry shown in Figs. 1(a) and 1(b). A superconducting region $S$ is connected to two normal electrodes $\mathrm{N}_{L}$ and $\mathrm{N}_{R}$ and to a superconducting electrode $S_{e}$, made of the same superconducting material as $S$. The three interfaces will be described by characteristic energies $\epsilon_{L}, \epsilon_{R}$, and $\epsilon_{S}$, defined below. As we are not interested in the spatial variation in the nonlocal correlations, we simplify the problem by assuming that the central superconducting region $S$ has dimensions smaller than the superconducting coherence length, which in the diffusive limit is given by $\xi_{S}=\sqrt{D / \Delta}$. Here $\Delta$ is the superconducting gap and $D$ is the diffusion coefficient. Thus, we may assume that the order parameter and the nonequilibrium distribution are uniform over $S .^{18}$

In order to determine either the nonlocal conductance [Fig. 1(a)] or resistance [Fig. 1(b)] we need to calculate the current density $\mathbf{j}$ and the self-consistent order parameter $\Delta$ in 
the $S$ region. As one can see from our results below, we always find a stationary current state. This behavior is distinctive for junctions consisting of a mesoscopic superconductor in contact with a bulk one. ${ }^{20}$ Thus, $\mathbf{j}$ and $\Delta$ can be expressed in terms of the Keldysh component of the matrix GF $\check{g}$

$$
\begin{gathered}
\Delta=\frac{\lambda}{4} \int d \epsilon \hat{g}_{12}^{K}, \\
\mathbf{j}=\frac{1}{8 e G_{N}} \int d \epsilon \operatorname{Tr}\left\{\hat{\tau}_{3} \check{g} \nabla \check{g}\right\}^{K},
\end{gathered}
$$

where $\lambda$ is the BCS coupling constant which determines the critical temperature, and $R_{N}$ is the normal state resistance of the $S$ region. The function $\check{g}$ is a $4 \times 4$ matrix in the $\mathrm{Nambu} \otimes$ Keldysh space with the usual structure

$$
\check{g}=\left(\begin{array}{cc}
\hat{g}^{R} & \hat{g}^{K} \\
0 & \hat{g}^{A}
\end{array}\right),
$$

while $\hat{g}$ are $2 \times 2$ matrices in Nambu space. In the diffusive limit these functions are the solutions of the Usadel equation $^{21}$

$$
-D \nabla(\check{g} \nabla \check{g})-i \epsilon\left[\hat{\tau}_{3}, \check{g}\right]-i[\Delta, \check{g}]=-i\left[\check{\Sigma}_{\mathrm{in}}, \check{g}\right],
$$

supplemented by the normalization condition $\check{g}^{2}=\check{1}$. Here $\check{\Sigma}_{\text {in }}$ is the self-energy term describing inelastic processes. In the time relaxation approach $\check{\Sigma}_{\text {in }}$ is proportional to $1 / \tau_{\text {in }}$, where $\tau_{\text {in }}$ is the inelastic relaxation time. We will assume that $1 / \tau_{\text {in }}$ is the smallest energy scale and neglect this term. At the interfaces with the electrodes we use the KupryianovLukichev boundary conditions ${ }^{22}$

$$
\left.D \check{g} \nabla \check{g}\right|_{\mathbf{n}}=\epsilon_{i} d\left[\check{g}, \check{g}_{i}\right],
$$

where $\check{g}_{i}$ are the GF of the electrodes $\left(i=L, R, S_{e}\right), \epsilon_{i}$ $=\epsilon_{\mathrm{Th}} / 2 r_{B}$, and $\epsilon_{\mathrm{Th}}=D / d^{2}$ is the Thouless energy, $r_{B}$ $=R_{B i} / R_{N}, R_{b i}$ is the $i$ th barrier resistance per unit area, and $\mathbf{n}$ denotes a unit vector normal to the interface. We assume that the GFs of the electrodes remain unchanged and equal to the bulk values, i.e., $\hat{g}^{R(A)}= \pm \hat{\tau}_{3}$ in the normal leads, and $\hat{g}_{S}^{R(A)}=g_{\mathrm{BCS}}^{R(A)} \hat{\tau}_{3}+f_{\mathrm{BCS}}^{R(A)} i \hat{\tau}_{2}$ in the superconductor electrode, where $g_{\mathrm{BCS}}^{R(A)}=\epsilon / \sqrt{(\epsilon \pm i \eta)^{2}-\Delta_{0}^{2}}$ and $f_{\mathrm{BCS}}^{R(A)}=\Delta_{0} / \sqrt{(\epsilon \pm i \eta)^{2}-\Delta_{0}^{2}}$. While the Keldysh components are given by

$$
\hat{g}_{i}^{K}=\hat{g}_{i}^{R} \hat{F}_{i}-\hat{F}_{i} \hat{g}^{A},
$$

where

$$
\hat{F}_{i}=F_{i+} \hat{\tau}_{0}+F_{i-} \hat{\tau}_{3},
$$

$V_{i}$ is the voltage in electrode $i$, and $F_{i \pm}$ $=\frac{1}{2}\left[\tanh \left(\frac{\epsilon+e V_{i}}{2 T}\right) \pm \tanh \left(\frac{\epsilon-e V_{i}}{2 T}\right)\right]$. We also assume that $V_{S_{e}}=0$. In principle the boundary conditions Eq. (5) are valid for low transmitting interfaces. In the present work we consider that the interface transparencies may vary in the range $10^{-3}$ $-10^{-1}$ for which Eq. (5) is sufficiently reliable.

With the help of Eq. (5) we can calculate the total current at each interface using the expression:

$$
e I_{i} R_{N}=\frac{\epsilon_{i}}{8 \epsilon_{\mathrm{Th}}} \int d \epsilon \operatorname{Tr} \tau_{3}\left[\check{g}_{i}, \check{g}\right]^{K} .
$$

In this case the GF inside $\mathrm{S}$ does not vary considerably and the Usadel Eq. (4) can be integrated over space coordinates using the boundary conditions Eq. (5). In this way one obtains a set of algebraic equations which can be written in a compact form

$$
[\check{\Lambda}, \check{g}]=0
$$

where

$$
\Lambda=\sum_{i=L, R, S} \epsilon_{i} \check{g}_{i}+\epsilon \tau_{3}+\check{\Lambda}-\check{\Sigma}_{\text {in }} .
$$

Equation (8) is equivalent to the Nazarov's circuit theory equations, ${ }^{23}$ which were used in Refs. 11 and 24 for nonlocal transport calculations. The solution for the R, A and $\mathrm{K}$ components of $\stackrel{g}{g}$ which satisfy Eq. (8) and the normalization condition can be formally be written as ${ }^{20,25}$

$$
\begin{gathered}
\hat{g}^{R(A)}=\frac{\hat{\Lambda}^{R(A)}}{\sqrt{\hat{\Lambda}^{R(A)} \hat{\Lambda}^{R(A)}}}, \\
\hat{g}^{K}=\frac{\hat{\Lambda}^{K}-\hat{g}^{R} \hat{\Lambda}^{K} \hat{g}^{A}}{\sqrt{\hat{\Lambda}^{R} \hat{\Lambda}^{R}}+\sqrt{\hat{\Lambda}^{A} \hat{\Lambda}^{A}}}
\end{gathered}
$$

Substituting these expressions into Eqs. (1) and (2) enable us to obtain numerically the self-consistent order parameter, the currents through the interfaces and the nonlocal voltage induced at the right electrode in the resistance measurement case.

\section{MEASUREMENT OF THE NONLOCAL CONDUCTANCE}

In this section we consider the experimental setup of Fig. 1(a). The left normal electrode is biased by a voltage source at $V_{L}$. For a nonlocal conductance measurement we will assume that the $N_{R}$ is grounded $\left(V_{R}=0\right)$, and compute the current $I_{R}$ through the interface $S / N_{R}$ from Eqs. (7), (9), and (10) and the self-consistent order parameter $\Delta$ from Eq. (1). Before we address the nonlocal properties of the system let us discuss the results concerning local properties.

In Fig. 2(a) we show the amplitude of $\Delta$ as a function of the bias voltage $V_{L}$ for $\epsilon_{S}=0.2 \Delta_{0}, \epsilon_{L}=0.1 \Delta_{0}$, and three different values of $\epsilon_{R}=0.01-0.1$, corresponding to transmission coefficients in the range $10^{-3}-10^{-2}$ (we assume that the length of the superconducting region is about $50 \mathrm{~nm}$ ). All energies are given in units of $\Delta_{0}$, which is the value of the order parameter in the bulk at $T=0$. For a fixed low value of $V_{L}, \Delta$ is reduced by increasing the coupling with the right normal electrode, which is a consequence of the inverse proximity effect. At some value $V_{L}^{*} \simeq 0.8 \Delta_{0}$ of the order of the self-consistent $\Delta$, one can see an abrupt reduction of the self-consistent order parameter. For voltages larger than $V_{L}^{*}$ the quasiparticle current through $S$ becomes considerably larger [Figs. 2(b) and 2(c)], i.e., the system is driven out of 

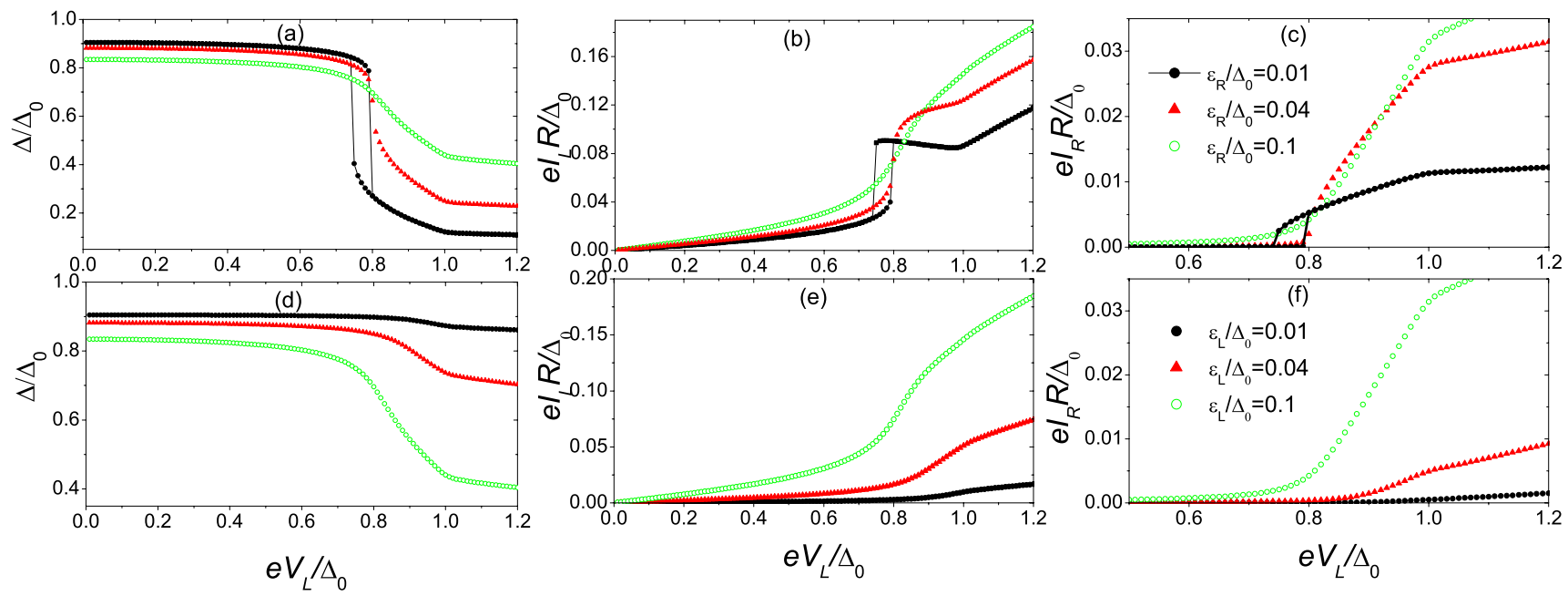

FIG. 2. (Color online) The amplitude of the self-consistent order parameter, the current $I_{L}$ injected from the left normal electrode and the current $I_{R}$ measured at the right electrode, as a function of the voltage $V_{L}$. Panels (a) - (c) for $\epsilon_{S}=0.2, \epsilon_{L}=0.1$, and $T=0.01 \Delta_{0}$ and different values of $\epsilon_{R}$. Panels (d)-(f) for $\epsilon_{S}=0.2, \epsilon_{R}=0.1$, and $T=0.01 \Delta_{0}$ and different values of $\epsilon_{L}$. We have defined $R=R_{N} \epsilon_{\mathrm{Th}} / \Delta_{0}$.

equilibrium. An interesting consequence of this nonequilibrium state for voltages $V_{L}>\sim V_{L}^{*}$ is the enhancement of the self-consistent gap by increasing the temperature, as shown in Fig. 3. This effect is related to the stimulation of superconductivity by quasiparticle currents in SIS systems and was studied both theoretical ${ }^{26}$ and experimentally. ${ }^{27}$

From Fig. 2(a) one can also see that the suppression of $\Delta$ at $V_{L}^{*}$ becomes more abrupt the weaker the coupling with the right electrode is. Figures 2(b) and 2(c) also show the corresponding current at the left and right interfaces. As expected the larger the resistance of the right interface (small $\epsilon_{R}$ ) the smaller the value of $I_{R}$. A strong nonequilibrium situation takes place when most of the current injected flows into the superconducting electrode, i.e., when $\epsilon_{R}$ is small enough (in our example $\left.\epsilon_{R}=0.01\right)$. In this case the gap becomes multivalued and this is reflected in the behavior of the currents $I_{L}$ and $I_{R}$. Multivalued solutions for the self-consistent gap were also found in Ref. 26 for SIS systems and recently in Ref. 28 for a NSN system.

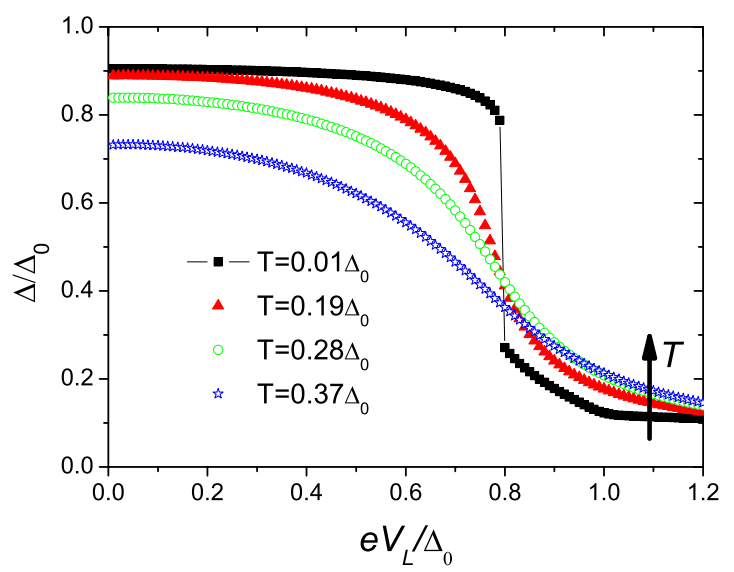

FIG. 3. (Color online) The amplitude of the self-consistent order parameter as a function of the voltage $V_{L}$ for different temperatures. Notice that in the range of temperatures shown and for large values of $V_{L}$ the gap is enhanced by increasing $T$.
Another interesting feature of this system is the existence of a region of voltages for which the local conductance is negative [see Fig. 2(b)]. This behavior was also obtained in Ref. 20 for a system consisting of a superconducting link separating a normal and a superconducting electrode. Also in Ref. 14 negative local conductance was obtained for a twodimensional ballistic superconductor attached to two normal electrodes. Notice however, that the part of the curve corresponding to a negative conductance would be not accessible in current biased experiments. If we now fix the value of $\epsilon_{R}$ at its maximum value $\epsilon_{R}=0.1$ and vary $\epsilon_{L}$, we see that even for the smallest coupling $\left(\epsilon_{L}=0.01 \Delta_{0}\right)$ the variation in the gap and the currents is smooth and no signatures of multivalued solutions appears for this range of parameters (see bottom row of Fig. 2).

Let us now focus on the nonlocal transport and compute the nonlocal conductance, which can be obtained easily from the knowledge of $I_{R}$ [Figs. 2(c) and 2(f)]. It is given by the expression

$$
G_{\mathrm{nl}}=\frac{\partial I_{R}}{\partial V_{L}}
$$

and shown in Fig. 4 as a function of the bias voltage $V_{L}$, for different values of the coupling energies $\epsilon_{L, R}$. In all cases $G_{\mathrm{nl}}$ is very small in the region of low voltages. This is in agreement with the zero nonlocal conductance obtained in the lowest order of tunneling ${ }^{8}$ due to the cancellation of the EC and CAR processes. However, our results are in all order of tunneling and therefore a dominance of EC (negative $G_{\mathrm{nl}}$ ) is obtained in accordance to Ref. 9. For voltages of the order of the self-consistent $\Delta$, the value of $G_{\mathrm{nl}}$ becomes significant. However, no change in sign is observed. As mentioned in the introduction, noninteracting models do not exhibit a change in sign of the nonlocal conductance in three-terminal NSN structures. This can only be achieved when electron-electron interactions are taken into account. ${ }^{16}$ 


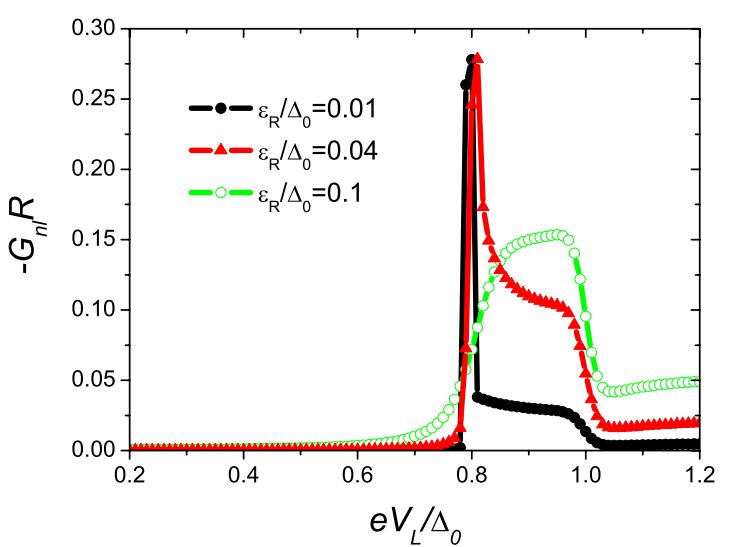

FIG. 4. (Color online) The voltage dependence of the (negative) nonlocal conductance $G_{\mathrm{nl}}$ normalized with respect to $R=R_{N} \epsilon_{\mathrm{Th}} / \Delta_{0}$. We have chosen $T=0.01 \Delta_{0}, \epsilon_{S}=0.2, \epsilon_{L}=0.1$, and $T=0.01 \Delta_{0}$ and different values of $\epsilon_{R}$.

\section{MEASUREMENT OF THE NONLOCAL RESISTANCE IN A THREE-TERMINAL DEVICE}

In a real experiment it is easier to measure a voltage rather than a current. Indeed the experiments of Refs. 1-5 were performed in (a) the current biased regime and (b) instead of the current, the nonlocal resistance (or voltage) was measured. Theoretically, it is not simple to impose a current bias. Therefore we will still work in the voltage biased case but determine the induced nonlocal voltage and resistance imposing zero current at the $S / N_{R}$ interface. Thus, all the current injected from the left normal electrode flows into the superconducting electrode $S_{e}$ [see Fig. 1(b)]. The current at the right interface is given by [cf. Eq. (7)]

$$
\begin{aligned}
e I_{R} R_{B R}= & \frac{1}{8} \int d \epsilon \operatorname{Tr} \hat{g}^{K}(\epsilon)-\frac{1}{4} \int d \epsilon \nu_{S}(\epsilon)\left(\tanh \left(\frac{\epsilon+e V_{R}}{2 T}\right)\right. \\
& \left.-\tanh \left(\frac{\epsilon-e V_{R}}{2 T}\right)\right)
\end{aligned}
$$

where $\nu_{S}(\epsilon)=\left(g^{R}-g^{A}\right) / 2$ is the density of states of $\mathrm{S}$ and $R_{B R}$ is the $R$ barrier resistance per unit area. The first term in the rhs is proportional to the quantity $Q^{*}$ identified in the literature as the charge imbalance potential, ${ }^{29}$ which appears due to a nonequilibrium distribution in the superconductor. The second term is the usual quasiparticle current term. The voltage $V_{R}$ is measured with respect to the ground [see Fig. 1(b)] and it is obtained by imposing $I_{R}=0$.

The results for $\Delta$, the injected current $I_{L}$ and the induced voltage $V_{R}$ as a function of the bias voltage $V_{L}$ are shown in Fig. 5, for fixed values of $\epsilon_{S, L}$ and different values of $\epsilon_{R}$ in the same range as in Fig. 2. In the case of low values of $\epsilon_{R}$, the self-consistent gap has a very similar behavior as in the preceding section. However, for the largest value $\epsilon_{R}=0.1 \Delta_{0}$ the suppression of $\Delta$ is larger as the one obtained by imposing $V_{R}=0$. Notice also that the region of negative local conductance [Fig. 5(b)], associated with the abrupt change in the order parameter appears now for all values of $\epsilon_{R}$. It is clear that by having imposed $I_{R}=0$ all the current injected must flow through the $S / S_{e}$ interface and our system behaves similarly to the $N / S / S$ studied in Ref. 20. In Fig. 5(c) we also show the voltage $V_{R}$ induced in the right electrode calculated by equalizing Eq. (12) to zero. At low $V_{L}$ values the induced voltage $V_{R}$ is very small, but it experiences a jump at the value of $V_{L}$ where the self-consistent gap exhibits its maximal drop.

We have now all quantities required to compute the nonlocal resistance which would be measured. This can be calculated from the expression

$$
R_{\mathrm{nl}}=\frac{\partial V_{X}}{\partial I_{L}}=\frac{\partial V_{X}}{\partial V_{L}}\left(\frac{\partial I_{L}}{\partial V_{L}}\right)^{-1}
$$

The measured voltage $V_{X}$ depends on the experimental setup. We are considering here the three-terminal structure of Fig. 1(b) and determining $V_{R}$ respect to the ground. Thus in this case $V_{X}=V_{R}$, i.e., the one shown in Fig. 5(c). According to Eq. (13) there are two factors determining the nonlocal resistance. One which is the inverse of the local conductance $G_{L L}^{-1}=d V_{L} / d I_{L}$ and which decrease by increasing $\epsilon_{L}$. The second contribution is given by $d V_{R} / d V_{L}$ which is nonzero only if a nonequilibrium distribution appears in the $S$ region and is
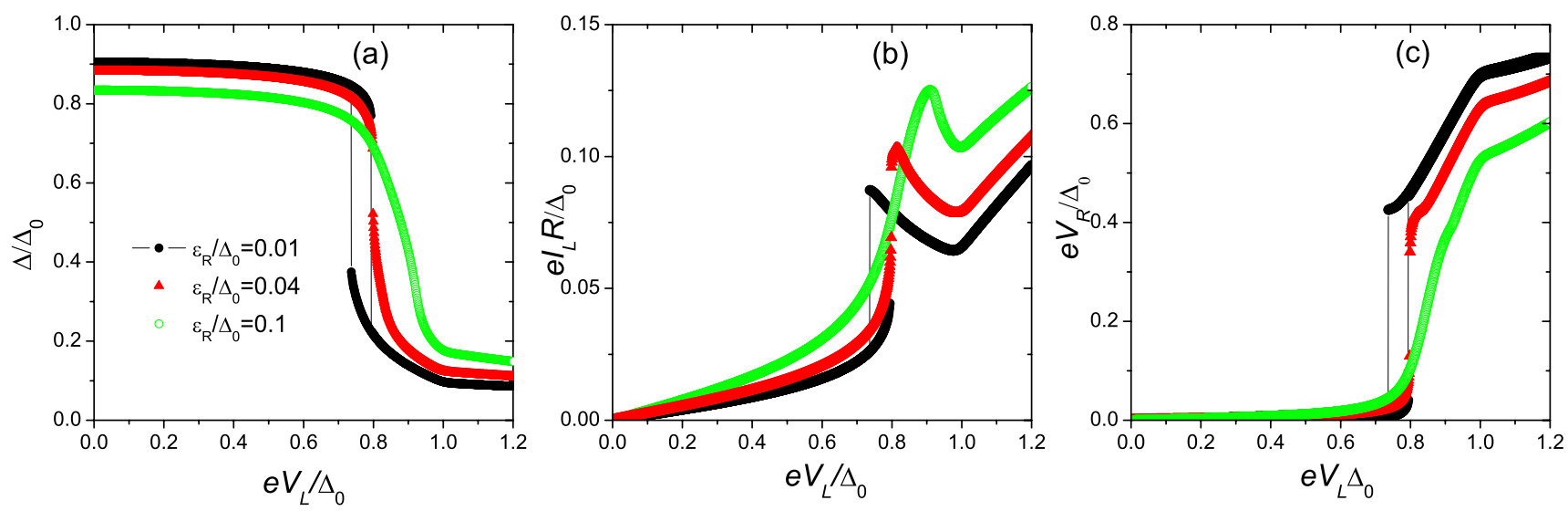

FIG. 5. (Color online) (a) The amplitude of the self-consistent order parameter, (b) the current $I_{L}$ injected from the left normal electrode, and (c) the induced voltage $V_{R}$ measured at the right electrode as a function of the voltage $V_{L}$ for $\epsilon_{S}=0.2, \epsilon_{L}=0.1$, and $T=0.01 \Delta_{0}$ and different values of $\epsilon_{R}$. 


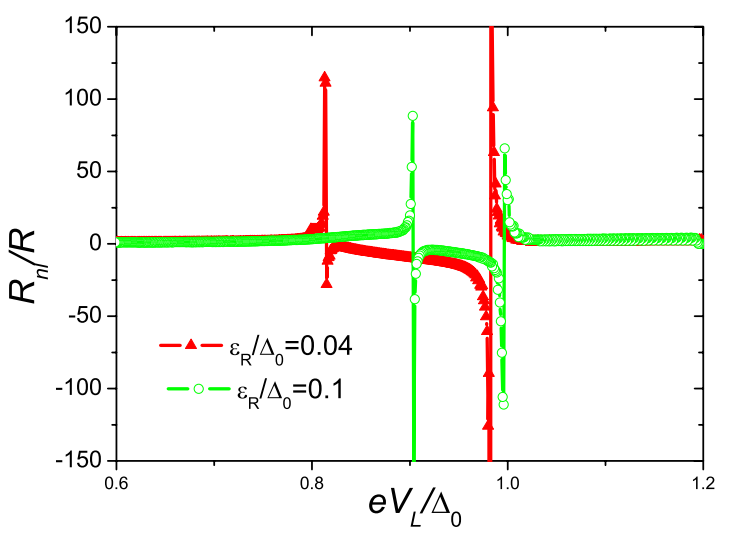

FIG. 6. (Color online) The voltage dependence of the nonlocal resistance calculated from Eq. (13) for $\epsilon_{S}=0.2 \Delta_{0}, \epsilon_{L}=0.1 \Delta_{0}$, and $T=0.01 \Delta_{0}$, and two values of $\epsilon_{R}$.

related to the charge imbalance term [cf. Eq. (12)].

In Fig. 6 we show the dependence $R_{\mathrm{nl}}\left(V_{L}\right)$ for two values of $\epsilon_{R}$ and $\epsilon_{L}=0.1 \Delta_{0}, \epsilon_{S}=0.2 \Delta_{0}$, and $T=0.01 \Delta_{0}$. The change in sign of $R_{\mathrm{nl}}$ is a consequence of the negative local conductance which appears between the values $V_{L} \simeq 0.8 \Delta_{0}$ and $V_{L}$ $\simeq \Delta_{0}$ [see Fig. 5(b)]. The position of the first peak of $R_{\mathrm{nl}}$ is determined by the value of $V_{L}$ at which $\Delta$ drops substantially, while the position of second peak is determine by $\Delta_{0}$. As mentioned above, in current bias experiments the curve $R_{\mathrm{nl}}$ may look very different to the ones shown in Fig. 6, since the region of negative local conductance may not be observed.

In experiments as those of Refs. 1, 3, and 5 the current flows along a superconducting wire, while in our model it flows into the reservoir $S_{e}$. For simplicity we have assume that the latter remains unaltered for all values $V_{L}$ considered here. In particular the value of the gap is the bulk BCS one for any value of $V_{L}$. However, in the experiments, when the current flowing through the wire reaches the critical value the superconducting gap is suppressed homogeneously in the region where the current is flowing. This leads to the observation of only one peak in the nonlocal resistance. ${ }^{3}$ In the next section we will model this situation.
We should also emphasize that the change in sign of the nonlocal resistance obtained in Fig. 6 is due to the fact that the local conductance $G_{L L}=d I_{L} / d V_{L}$ becomes negative for some values of $V_{L}$ [cf. Fig. 5(b)]. If the coupling $\epsilon_{S}$ is large enough, the local conductance remains always positive and so the nonlocal resistance. This is shown in Fig. 7, where the amplitude of the self-consistency gap, the current injected, and the voltage induced at the right electrode are plotted as a function of $V_{L}$, for $\epsilon_{S}=1,1.5$ and $2 \Delta_{0}$. If one compares these results with those obtained for a smaller $\epsilon_{S}$ (Fig. 5), one sees that $\Delta$ is now only weakly suppressed and that the current $I_{L}$ increases monotonically. Thus the nonlocal resistance is always positive as it is shown in Fig. 8.

Finally, we show in Fig. 9 the temperature dependence of the zero bias nonlocal resistance for different values of the coupling parameter $\epsilon_{S}$. We can see that while for the small values of $\epsilon_{S}$ the nonlocal resistance increases monotonously with the temperature, for larger values of $\epsilon_{S}, R_{\mathrm{nl}}$ reaches a maximum value. In the latter case the charge imbalance effect becomes important and dominates over the local conductance factor for large temperatures. This behavior is in agreement with previous calculations of $R_{\mathrm{nl}}$ in a superconducting quantum dot. ${ }^{13}$ One could conclude as in Ref. 15 that the nonmonotonic behavior of $R_{\mathrm{nl}}(T)$ is in qualitative agreement with the observations of Refs. 1 and 3 . However, we hardly believe that. If this would be the case then one should obtain for the same parameter range a change in sign for $R_{\mathrm{nl}}$ as a function of the injected current, as observed in the experiments. ${ }^{3}$ On the contrary, Fig. 9 clearly shows a monotonic increase in $R_{\mathrm{nl}}$ with the applied voltage. In the next section we will show that the change in sign of $R_{\mathrm{nl}}$ observed in the experiment is due to the suppression of the superconductivity by the injection of a current. Also the peak of $R_{\mathrm{nl}}$ observed as a function of temperature could be understood within this model.

\section{FOUR-TERMINAL STRUCTURE}

We now consider a situation closer to that of the experiments of Refs. 3 and 5, in which the nonlocal resistance
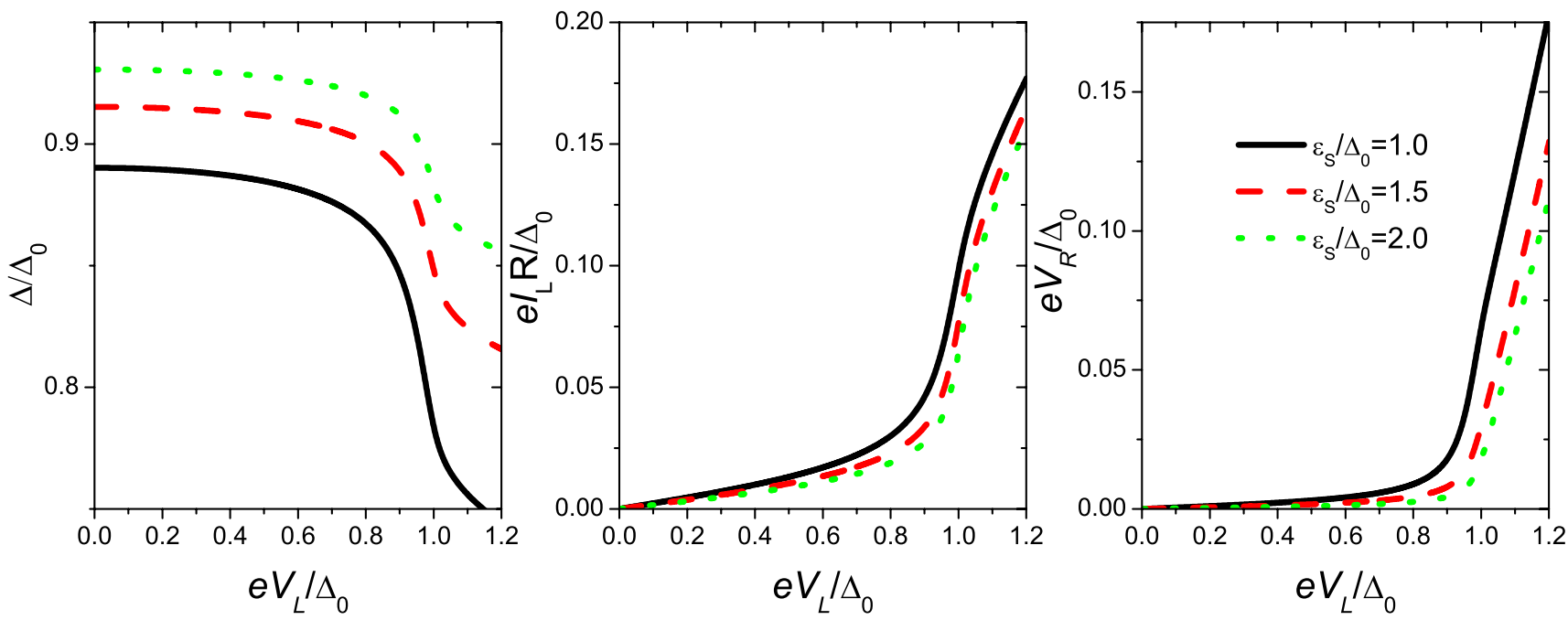

FIG. 7. (Color online) The amplitude of the self-consistent order parameter, the current $I_{L}$ injected from the left normal electrode and the induced voltage $V_{R}$ measured at the right electrode, as a function of the voltage $V_{L}$, for $\epsilon_{L}=\epsilon_{R}=0.1, T=0.01 \Delta_{0}$, and different values of $\epsilon_{S}$. 


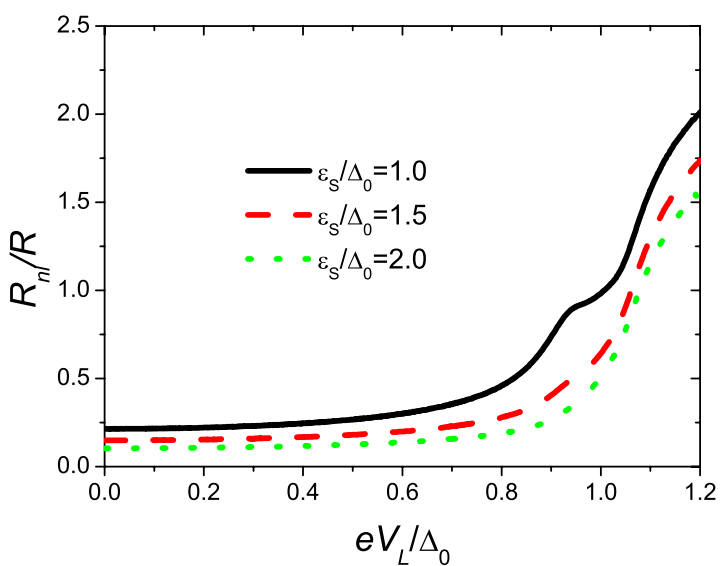

FIG. 8. (Color online) The voltage dependence of nonlocal resistance for the same parameters as in Fig. 7.

(voltage) has been measured in a multiterminal setup consisting of a superconducting wire attached to several normal terminals. In these experiments the nonlocal voltage corresponds to the potential difference between one end of the superconducting wire and one of the normal leads. We model these experimental situations as shown in Fig. 10. On the top of a superconducting wire we place two normal contacts. Current flows from the $N_{L}$ contact to the $N_{e}$ due to the bias voltage $V_{L}$ applied between the contacts. We are interested in the voltage difference measured between the end of the wire, which we denote by $S^{\prime}$, and an additional normal contact $N_{R}$. We use on purpose the same notation as in Fig. 1 in order to use straightforwardly the expressions derived in Sec. II. The only difference is that the drain electrode is now in the normal state and that we measure the voltage difference between the end $S^{\prime}$ of the superconducting slab and the normal electrode $N_{R}$. The electrode $N_{e}$ is grounded. In order to compute the resistance measured between $S^{\prime}$ and $N_{R}$ we proceed as in the last section, determining the Green functions from Eqs. (9) and (10) and the self-consistent gap. Now we impose that the currents through the $S / N_{R}$ and through the $S / S^{\prime}$ interfaces are zero. The current trough the $S / S^{\prime}$ interface can be written as the sum

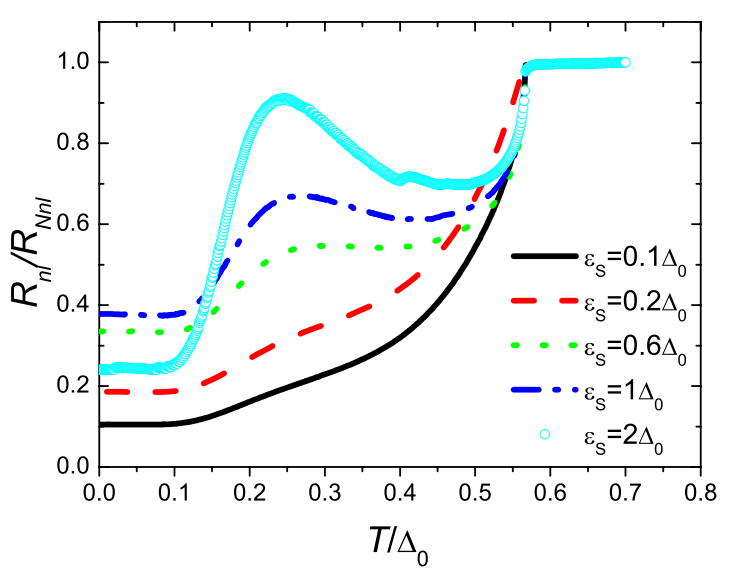

FIG. 9. (Color online) The temperature dependence of the zero bias nonlocal resistance measure in a three-terminal device for different values of $\epsilon_{S}$. We have chosen $\epsilon_{L}=\epsilon_{R}=0.1 \Delta_{0}$.

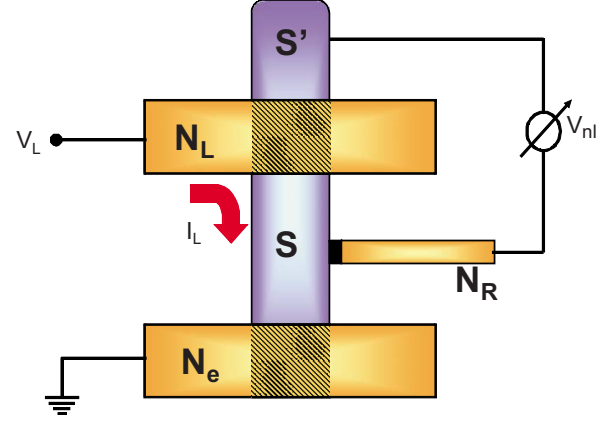

FIG. 10. (Color online) Sketch of the four-terminal structure under consideration.

$$
I_{S / S^{\prime}}=I_{J}+I_{q p}
$$

of the Josephson and the quasiparticle contribution, respectively. The first is given by the product of anomalous Green's functions in Eq. (2) while the latter by the product of normal components. Since no current is flowing into $S^{\prime}$ we assume that there the Green's functions are those in equilibrium, with $|\Delta|$ equal to the self-consistent $|\Delta|$ in the $S$ region at $V_{L}=0$, and a phase $\phi$ which is determined by imposing the condition of no current through the $S / S^{\prime}$ interface. For voltages lower than a critical voltage $V_{L}^{*} \sim \Delta$ we always found a finite value of $\phi$. In this case the voltage $V^{\prime}$ induced in $S^{\prime}$ equals zero, and the measured $V_{\mathrm{nl}}$ coincides with $V_{R}$ of the previous section. The voltage $V_{L}^{*}$ is the voltage at which the self-consistent gap vanishes, i.e., when the current flowing in $S$ reaches its critical value. For values of $V_{L}$ larger than this value the quasiparticle current becomes finite, and a voltage $V^{\prime}$ is induced in $S^{\prime}$. We compute it from an expression obtained by equalizing Eq. (7) to zero. Thus, the nonlocal resistance is given by Eq. (13) where now $V_{X}=V_{R}$ for $V_{L}$ $<V_{L}^{*}$ and $V_{X}=V_{R}-V^{\prime}$ for $V_{L}>V_{L}^{*}$. In Fig. 11 we show the result of our calculation for $R_{\mathrm{nl}}$ as a function of $V_{L}$. It shows a peak at $V_{L}^{*}$. Since the latter is of the order of $\Delta$, the peak is shifted to lower voltages by increasing the temperature (Fig. 11). This behavior is in agreement with the experimental

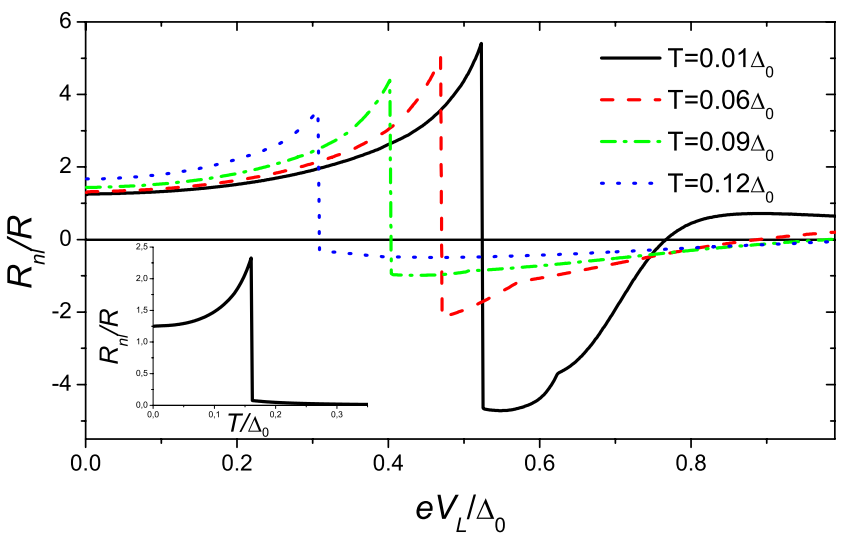

FIG. 11. (Color online) The nonlocal resistance measured in a four terminal structure as a function of the bias voltage $V_{L}$ for $\epsilon_{L}$ $=\epsilon_{R}=0.1 \Delta_{0}, \epsilon_{S}=0.2$, and different values of the temperature. Inset: the nonlocal resistance as a function of the temperature at zero bias. 
observation of Ref. 3, where the peak occurred at values of the bias current close to the value of critical current of the superconducting wire. The change in sign of $R_{\mathrm{nl}}$ is related to the nonequilibrium situation created in $S$ by the injection of a current from $N_{L}$. We also show in the inset of Fig. 11 the temperature dependence of $R_{\mathrm{nl}}$, which exhibits a pronounced peak for $V_{L} \simeq V_{L}^{*}$. This is again in agreement with the observations of Ref. 3. These results demonstrate that the model studied here contains the main ingredients for describing experiments on nonlocal transport as the one of Ref. 3. Within this model the change in sign of the nonlocal resistance has its origin in the deviation of the distribution function of the superconductor from the equilibrium one. Notice, that as in Ref. 3, the change in sign of $R_{\mathrm{nl}}$ occurs at the critical current which corresponds to the voltage $V_{L}^{*}$ in our model.

In other experiments, ${ }^{2,5}$ however, the change in sign occurred at lower voltages. This discrepancy is at the moment not clarified and may be related to the inclusion of electronelectron interactions as proposed in Ref. 16.

\section{CONCLUSIONS}

We have presented a self-consistent analysis of the transport properties of a structure consisting of a mesoscopic superconductor whose dimensions are smaller than the characteristic length $\xi_{S}$, attached to two normal and one superconducting terminals. We have analyzed two measurement methods: one in which the detector $(\mathrm{R})$ electrode is grounded and the leaking current is measured, and one in which the current through this lead is fixed to zero and the induced voltage is measured. In both cases we observe that the self-consistent order parameter in the mesoscopic central region exhibits an abrupt drop at a certain voltage of the order of the self-consistent $\Delta$. Associated to this drop the local differential conductance at the injector lead (L) may become negative for certain values of the coupling parameters, resulting in a change in sign of the nonlocal resistance. As we stress throughout this manuscript, this change in sign would not be related to a dominance of CAR over EC processes but to a nonequilibrium effect. We have still described another mechanism for the appearance of negative nonlocal resistance which is probably most suitable for explaining the observations of Ref. 3. This mechanism is applicable in a four terminal geometry and corresponds to the injection of large currents which may switch the superconducting region into the normal state. The observation of a change in sign in the nonlocal signal at smaller bias and small transparencies like the ones reported in Refs. 2 and 5 are certainly not possible to be explained with the theoretical model presented in this work and might be related to the influence of electronelectron interactions, as already pointed out in Ref. 16. Further work for analyzing the combined effect of interactions and nonequilibrium effects is under progress.

\section{ACKNOWLEDGMENTS}

We thank R. Mélin for useful reading of the manuscript. Financial support from Spanish MICINN under Contracts No. FIS2005-06255 and No. FIS2008-04209 is acknowledged. F.S.B. acknowledges funding by the Ramón y Cajal program.
${ }^{1}$ D. Beckmann, H. B. Weber, and H. v. Löhneysen, Phys. Rev. Lett. 93, 197003 (2004).

${ }^{2}$ S. Russo, M. Kroug, T. M. Klapwijk, and A. F. Morpurgo, Phys. Rev. Lett. 95, 027002 (2005).

${ }^{3}$ P. Cadden-Zimansky and V. Chandrasekhar, Phys. Rev. Lett. 97, 237003 (2006); P. Cadden-Zimansky, Z. Ziang, and V. Chandrasekhar, New J. Phys. 9, 116 (2007).

${ }^{4}$ D. Beckmann and H. v. Löhneysen, Appl. Phys. A: Mater. Sci. Process. 89, 603 (2007)

${ }^{5}$ A. Kleine, A. Baumgartner, J. Trbovic, and C. Schönenberger, EPL 87, 27011 (2009)

${ }^{6}$ A. F. Andreev, Zh. Eksp. Teor. Fiz. 46, 1823 (1964) [Sov. Phys. JETP 19, 1228 (1964)].

${ }^{7}$ J. M. Byers and M. E. Flatté, Phys. Rev. Lett. 74, 306 (1995).

${ }^{8}$ G. Falci, D. Feinberg, and H. Hekking, Europhys. Lett. 54, 255 (2001).

${ }^{9}$ R. Mélin and D. Feinberg, Phys. Rev. B 70, 174509 (2004); S. Duhot and R. Mélin, Eur. Phys. J. B 53, 257 (2006); R. Mélin, Phys. Rev. B 73, 174512 (2006).

${ }^{10}$ M. S. Kalenkov and A. D. Zaikin, Pis'ma Zh. Eksp. Teor. Fiz. 87, 166 (2008) [JETP Lett. 87, 140 (2008)].

${ }^{11}$ J. P. Morten, A. Brataas, and W. Belzig, Phys. Rev. B 74, 214510 (2006).

${ }^{12}$ A. Brinkman and A. A. Golubov, Phys. Rev. B 74, 214512 (2006).
${ }^{13}$ D. S. Golubev and A. D. Zaikin, Phys. Rev. B 76, 184510 (2007).

${ }^{14}$ R. Melin, F. S. Bergeret, and A. Levy Yeyati, Phys. Rev. B 79, 104518 (2009).

${ }^{15}$ D. S. Golubev, M. S. Kalenkov, and A. D. Zaikin, Phys. Rev. Lett. 103, 067006 (2009).

${ }^{16}$ A. Levy Yeyati, F. S. Bergeret, A. Martin-Rodero, and T. M. Klapwijk, Nat. Phys. 3, 455 (2007).

${ }^{17}$ M. S. Kalenkov and A. D. Zaikin, Phys. Rev. B 75, 172503 (2007).

${ }^{18}$ For low temperatures and voltages, nonlocal correlations are governed by EC and CAR processes; i.e., their characteristic decay length is $\xi_{S}$, as shown in Ref. 19. For higher temperatures or currents charge imbalance effects may become important. The length controlling the charge imbalance, though, is larger than $\xi_{S}$ and therefore our assumption remains valid.

${ }^{19}$ D. Feinberg, Eur. Phys. J. B 36, 419 (2003).

${ }^{20}$ A. V. Zaitsev, A. F. Volkov, S. W. D. Bailey, and C. J. Lambert, Phys. Rev. B 60, 3559 (1999).

${ }^{21}$ K. D. Usadel, Phys. Rev. Lett. 25, 507 (1970).

${ }^{22}$ M. Kupriyanov and V. F. Lukichev, Zh. Eksp. Teor. Fiz. 94, 139 (1988) [Sov. Phys. JETP 67, 1163 (1988)].

${ }^{23}$ Yu. V. Nazarov, Superlattices Microstruct. 25, 1221 (1999).

${ }^{24}$ J. P. Morten, D. Huertas-Hernando, W. Belzig, and A. Brataas, Phys. Rev. B 78, 224515 (2008). 
${ }^{25}$ A. V. Zaitsev, Pis'ma Zh. Eksp. Teor. Fiz. 51, 35 (1990) [JETP Lett. 51, 41 (1990)].

${ }^{26}$ A. V. Zaitsev, Pis'ma Zh. Eksp. Teor. Fiz. 55, 66 (1992) [JETP Lett. 55, 67 (1992)].

${ }^{27}$ M. G. Blamire, E. C. G. Kirk, J. E. Evetts, and T. M. Klapwijk,
Phys. Rev. Lett. 66, 220 (1991).

${ }^{28}$ I. Snyman and Yu. V. Nazarov, Phys. Rev. B 79, 014510 (2009).

${ }^{29}$ J. Clarke, Phys. Rev. Lett. 28, 1363 (1972); M. Tinkham and J. Clarke, ibid. 28, 1366 (1972). 